\title{
Transparent lattice characterization with gated turn-by-turn data of diagnostic bunch train
}

\author{
Yongjun Li, Weixing Cheng, Kiman Ha, and Robert Rainer \\ Brookhaven National Laboratory, Upton, New York 11973, USA
}

(Received 19 July 2017; published 21 November 2017)

\begin{abstract}
Methods of characterization of a storage ring's lattice have traditionally been intrusive to routine operations. More importantly, the lattice seen by particles can drift with the beam current due to collective effects. To circumvent this, we have developed a novel approach for dynamically characterizing a storage ring's lattice that is transparent to operations. Our approach adopts a dedicated filling pattern which has a short, separate diagnostic bunch train (DBT). Through the use of a bunch-by-bunch feedback system, the DBT can be selectively excited on demand. Gated functionality of a beam position monitor system is capable of collecting turn-by-turn data of the DBT, from which the lattice can then be characterized after excitation. As the DBT comprises only about one percent of the total operational bunches, the effects of its excitation are negligible to users. This approach allows us to localize the distributed quadrupolar wakefields generated in the storage ring vacuum chamber during beam accumulation. While effectively transparent to operations, our approach enables us to dynamically control the beta beat and phase beat, and unobtrusively optimize performance of the National Synchrotron Light Source-II accelerator during routine operations.
\end{abstract}

DOI: 10.1103/PhysRevAccelBeams.20.112802

\section{INTRODUCTION}

For high brightness synchrotron light sources, it is essential to mitigate lattice distortion to optimize performance during routine operations. At National Synchrotron Light Source-II (NSLS-II) [1], the deviation of the linear lattice has been observed but not quantitatively characterized during operations. Unlike orbit drift, which can be directly monitored by beam position monitors (BPMs) and then controlled by an orbit correction and/or feedback system, dynamic lattice characterization is more difficult to perform without interfering with operations. Undetected lattice distortion can degrade global machine performance over time. This can lead to poor injection efficiency, decreased brightness, reduced beam lifetime, etc.

Although there are several methods to characterize and correct the linear lattice during dedicated machine studies periods, they often interfere with stable beam conditions. At NSLS-II, a credited safety system known as the active interlock system (AIS) is engaged to constrain the stored beam within a narrow window, at currents above $2 \mathrm{~mA}$. The AIS is in place to avoid possible hardware or equipment damage and is a subsystem of the global equipment protection system (EPS). Many lattice characterization

\footnotetext{
yli@bnl.gov
}

Published by the American Physical Society under the terms of the Creative Commons Attribution 4.0 International license. Further distribution of this work must maintain attribution to the author(s) and the published article's title, journal citation, and DOI. methods would violate the AIS, making it impractical to implement them during operations. Safety systems aside, however, beam manipulation of the magnitude that some lattice characterization techniques require would interfere with beam line experiments that have high sensitivity. Common tools used for lattice characterization and/or correction include, but are not limited to linear optics from closed orbit (LOCO) [2,3], turn-by-turn (TbT) data of a short bunch train excited by a short pulse excitation [4-9], or a long bunch train excited by the bunch-by-bunch feedback system [10].

One of the more common tools for lattice characterization is the use of the LOCO method. While this technique is reliable, it requires changing all correctors throughout the storage ring individually and sequentially, and measuring the orbit response matrix (ORM). Measuring the ORM and implementing the corresponding parameter fitting can be time consuming for large scale rings. LOCO measurements also require continuous beam perturbation, which detracts from usable, stable beam time. At NSLS-II specifically, beam manipulation necessary for LOCO measurements could potentially perturb the beam outside of the AIS window. As the nominal operating current is presently $300 \mathrm{~mA}$, violation of the AIS would result in a beam dump, interrupting operations.

With modern advancements in BPM technology, storage ring lattices can be characterized with accurately aligned BPM turn-by-turn (TbT) data. To accomplish this, the beam is excited with a pulsed magnet, also known as a "pinger" magnet. At most light source facilities, however, the pulse width of a pinger wave usually lasts several microseconds, 
while the separation between two adjacent buckets is a few nanoseconds. If one were to use the pinger pulse to perturb the beam of a long bunch train, each bunch would experience a different excitation depending on its arrival time. Due to a limited bandwidth of the bandpass filter, most of the existing BPM systems are unable to resolve the bunch-by-bunch signals. The TbT data that reveals the centroid motion of the long bunch train is therefore highly decoherent after excitation [11-13], making it unsuitable for accurate lattice characterization. To obtain clean TbT data with such a long pulse width, one would need to utilize a shorter bunch train to minimize the disparity in arrival times of the bunches. Or one could utilize a well designed pulsed magnet with a wide flattop waveform [14], and therefore minimize the excitation deviation. During operations, storage rings are filled with long bunch trains to achieve higher stored beam current. Lattices seen by the beam at high operational current differ from ones at low current due to the wakefields generated in the vacuum chamber and therefore, the pinger excitation is not capable of generating valid data for a long bunch train. In addition, the pinger excitation necessary for lattice characterization would disturb stable beam conditions and possibly violate the AIS.

A method of exciting long bunch trains with a bunch-bybunch feedback system (BBFB) has been developed at Diamond light source [10]. The excitation amplitudes are small (significantly less than the beam size) but at high frequencies. Their method involves collecting the TbT data with dedicated signal processing, after excitation. To characterize the lattice during operations, however, requires excitation of the whole operational bunch train. To achieve comparable resolution as the pinger excitation technique, their method also requires continuous excitation of the whole operational bunch train. Our method introduces a more transparent technique for lattice characterization that utilizes a short diagnostic bunch train (DBT) developed at NSLS-II [15]. The DBT is isolated from the main user bunch train and transversely excited with the BBFB system [16]. The TbT data of the DBT is collected using the gated functionality of BPMs. As the DBT (ten bunches) comprises only about one percent of the total bunches, and the excitation amplitude is less than 1 millimeter, the effects on the global beam motion are negligible to users. Additionally, an "on-demand" triggering mode is utilized to minimize the disturbance on the circulating beam. With minimal beam disturbance, this approach is effectively transparent to the beam lines and can be applied at any time during operations without interfering with experiments, even ones requiring high sensitivity.

The method for transparent lattice characterization has been developed and demonstrated at NSLS-II, a state-ofthe-art third generation light source in operation at Brookhaven National Laboratory. Its main parameters
TABLE I. Main parameters of NSLS-II's ring.

\begin{tabular}{lc}
\hline \hline Parameters & Values \\
\hline Circumference $(\mathrm{m})$ & 792 \\
Number of DBA cell & 30 \\
Tune & $33.22 / 16.26$ \\
rf frequency $(\mathrm{MHz})$ & 499.68 \\
Harmonic number & 1320 \\
Horizontal emittance (nm rad) & $2.2(\mathrm{w} / \mathrm{o} \mathrm{DWs})$ \\
Horizontal emittance (nm rad) & $1.0(\mathrm{w} / \mathrm{DWs})$ \\
Vertical emittance $(\mathrm{pm} \mathrm{rad})$ & 8 \\
\hline \hline
\end{tabular}

are listed in Table I. As a third generation light source, requirements for brightness and stability are challenging and demanding. NSLS-II lattice has 30 double bend achromatic (DBA) cells. Two DBA cells with mirror symmetry inside have high- and low- $\beta_{x}$ functions at short and long straight sections respectively. The whole lattice has 15-fold symmetry. Three damping wigglers (DWs) are included to reduce the horizontal emittance from $2.2 \mathrm{~nm}$ rad to $1.0 \mathrm{~nm}$ rad. The threefold linear lattice with DWs is designed to be approximately 15 -fold symmetric.

To further explain the development of the transparent lattice characterization technique and its application to NSLS-II, the remaining sections are outlined as follows: Section II describes the techniques of exciting an isolated DBT selectively and collecting its TbT data with the gated functionality of BPMs. Section III introduces the methods used to characterize the lattice based on TbT data. The strategy of using a weighted linear response matrix to correct the lattice is also explained. In Sec. IV, we applied our new approach to characterize the linear lattice during beam current accumulation at NSLS-II. From the lattice dependence on the beam current, the quadrupolar wakefields can be localized around the ring and lattice correction can be implemented systematically. A brief summary and a short discussion on the future BPM technology development will be given in Sec. V.

\section{SELECTIVE BUNCH EXCITATION AND GATED TBT DATA ACQUISITION}

In this section we discuss the necessary requirements for lattice characterization by designing a technique which utilizes a dedicated filling pattern configuration, gated bunch-train excitation and data acquisition.

\section{A. Diagnostic bunch train (DBT)}

During routine operation of the NSLS-II storage ring, various instabilities due to collective effects are suppressed by the transverse BBFB system, allowing the ring to operate at high beam current. High precision lattice characterization, however, requires beam excitation which the BBFB would normally prevent. To bypass this, a short DBT is filled and is separated from the main, long 


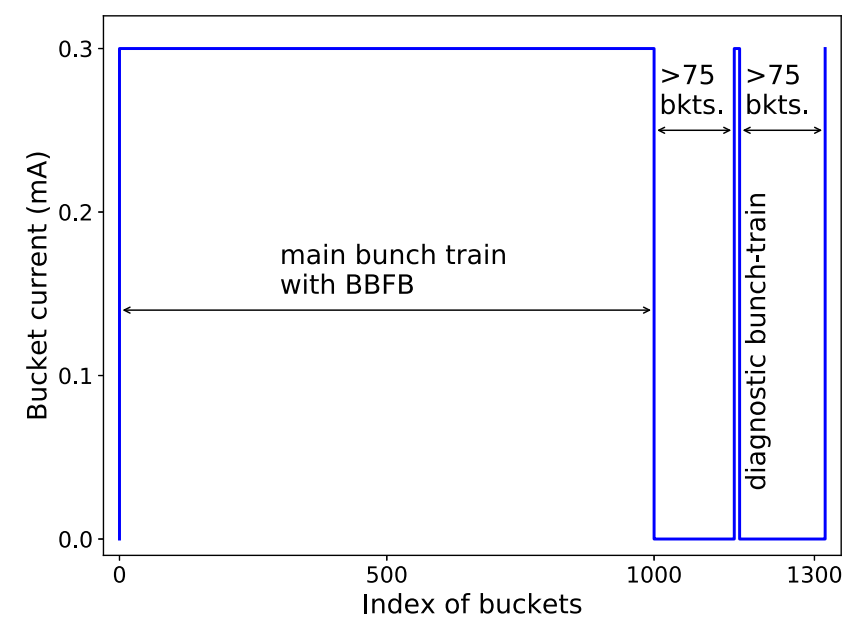

FIG. 1. Bucket filling pattern with an extra DBT for transparent lattice characterization.

operational bunch train (see Fig. 1). The BBFB can be configured to only stabilize the operational bunch train, and not the DBT. In this configuration the excited betatron motion of the DBT is not suppressed while the main bunch train remains stable. At NSLS-II, the isolated DBT has been found stable even without the use of feedback on it. Although our method for lattice characterization requires the BBFB to not operate on the DBT, it is possible to configure the BBFB system to be functional for the DBT when excitation is not taking place. In the event of excitation of the DBT, the BBFB can be switched off (for the DBT only) for the short period of time $(\sim \mathrm{ms})$ needed for data acquisition and then reestablished immediately after. The separation between the DBT and the main bunch train(s) needs to be larger than 75 empty buckets ( $\sim 150 \mathrm{~ns}$ ) due to the $\pm 10 \mathrm{MHz}$ bandwidth of the bandpass filter, which will be covered in greater detail later.

During normal operations, the BPM system needs to deliver both $10 \mathrm{~Hz}$ data for slow orbit monitoring and $10 \mathrm{kHz}$ data for fast orbit feedback at all times. The $10 \mathrm{~Hz}$ and $10 \mathrm{kHz}$ data need to be ungated to include all the bunch signals and the radio frequency (rf) attenuation on the BPMs set to correspond to the beam current. To obtain clean $\mathrm{TbT}$ signals with good signal-to-noise ratio the charge of the DBT needs to be maintained at a level of $\sim 1 \%$ of the total charge of the operational bunches. The DBT is comprised of $1 \%$ of the total operational bunches. Because the charge of the buckets on the DBT is comparable to that of the charge in the operation bunches, they experience similar wakefields and external excitations.

\section{B. Selective bunch excitation}

The selective bunch excitation on the DBT is accomplished with the BBFB system. The digitizer of the NSLS-II BBFB system has an integrated function that can excite any selected bunches [17]. To do so, the precise

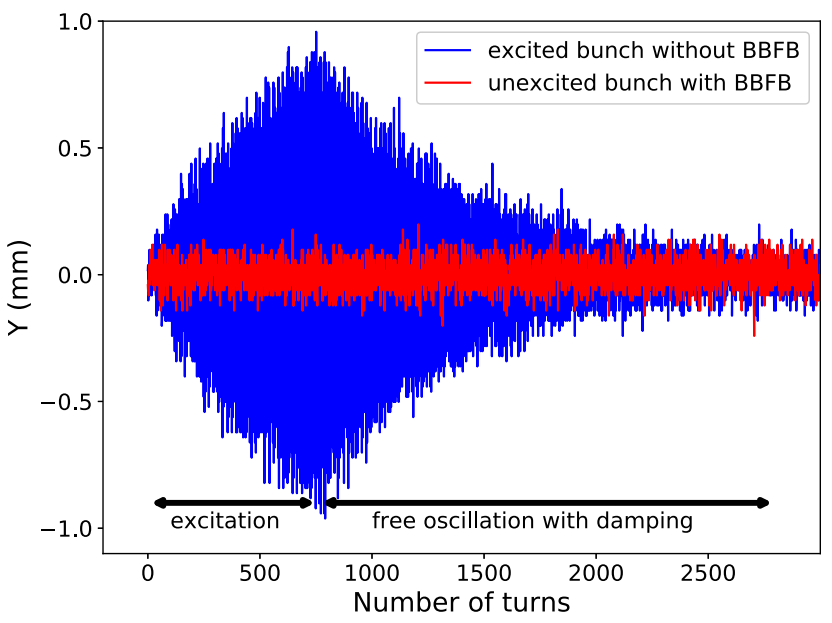

FIG. 2. Analog-to-digital converter (ADC) signals (counts) from BPM buttons of an excited bunch (in blue) without BBFB suppression and an unexcited bunch (in red) with BBFB suppression. Excitation is performed through resonance driving for about 700 turns $(\leq 2 \mathrm{~ms})$. Free betatron oscillation then decays through radiation damping.

betatron tune of the diagnostic bunches is determined by sweeping across a frequency range to reveal their resonant peaks. The diagnostic bunches can then be excited at their resonance frequencies with the BBFB system. It typically takes less than $2 \mathrm{~ms}$ for the betatron amplitude to reach about $1 \mathrm{~mm}$ (see Fig. 2). The excitation trigger can be configured for an external, on-demand mode. The same trigger can then be synchronized with the BPM gated data acquisition, which will be elaborated on later.

There are several bunches in the DBT which need to be excited in phase. Excitation is performed through resonance driving of the DBT bunches. An out-of-phase excitation within the bunch train can lead to an amplitude decoherence, corrupting its TbT data. The DBT is excited at the first harmonic of the betatron oscillation frequency. As long as the bunches have betatron frequencies that are close, the excited bunch motions will be in phase. There is one dedicated BPM used as the digital bunch-by-bunch feedback's pickup, which can distinguish different bunches in the train. Measuring the TbT data of the different bunches in the DBT with this dedicated BPM reveals centroid data that is consistent with our expected results (see Fig. 3).

\section{Gated TbT data acquisition}

The gated BPM data acquisition is accomplished through in-house BPM technology developed at NSLS-II. It includes two boards: analog front-end (AFE) and digital front-end (DFE). The AFE has a bandpass filter with a center frequency of $500 \mathrm{MHz}$ and a bandwidth of $\pm 10 \mathrm{MHz}$, which determines the impulse response of $\sim 200 \mathrm{~ns}(\sim 25$ ADC samples at $117 \mathrm{MHz}$ sampling rate). The DFE board processes the sampled raw ADC information to deliver TbT, $10 \mathrm{kHz}$ and $10 \mathrm{~Hz}$ data and sends them to the controls 


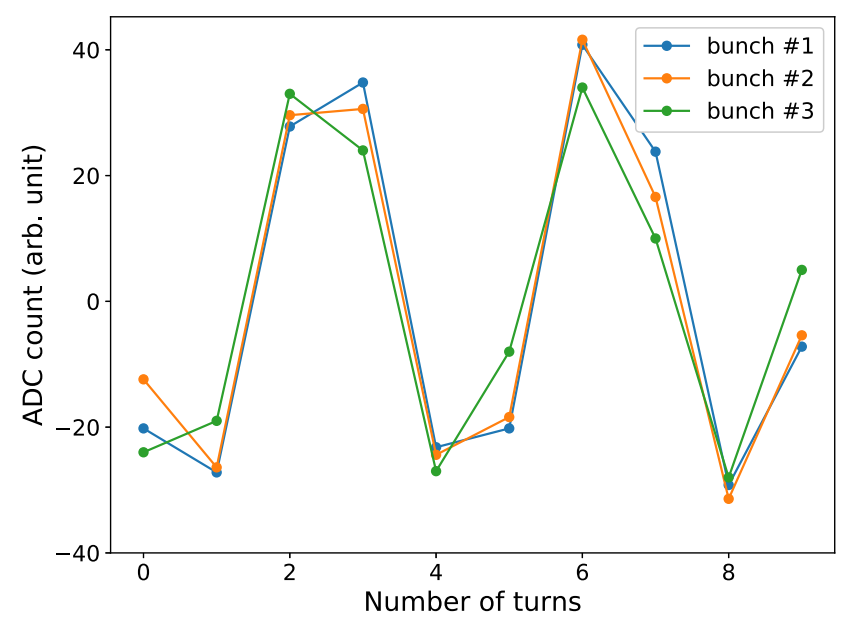

FIG. 3. In-phase excitation of the DBT through resonance driving. Here the vertical ADC counts of three out of ten bunches are shown. The signals are measured by the dedicated BPM used as the BBFB's pickup, and its digitizer can distinguish different bunches in the train.

network. Detailed information of NSLS-II BPM electronics developments can be found in [18]. The $\pm 10 \mathrm{MHz}$ bandpass filter and $117 \mathrm{MHz}$ sampling rate render NSLS-II BPM electronics incapable of resolving the bunch-to-bunch positions. If the diagnostics bunches are separated by more than $150 \mathrm{~ns}$, the digitizer is then capable of resolving them. The DBT must therefore be separated from the main operational bunch trains by at least 75 empty buckets from both sides (see Fig. 1). The gated functionality of the BPMs has been implemented inside the field-programmable gate array. The schematic diagram of the gated signal processing is shown in Fig. 4. Two signal-processing channels with separated gates are provided. The delay and width of each gate can be adjusted independently so that signals from different bunch trains can be selected and processed separately and simultaneously. One of the channels (Gate 2) can then be dedicated to lattice characterization. Expanding the number of channels could allow processing of multiple DBTs for possible future use.

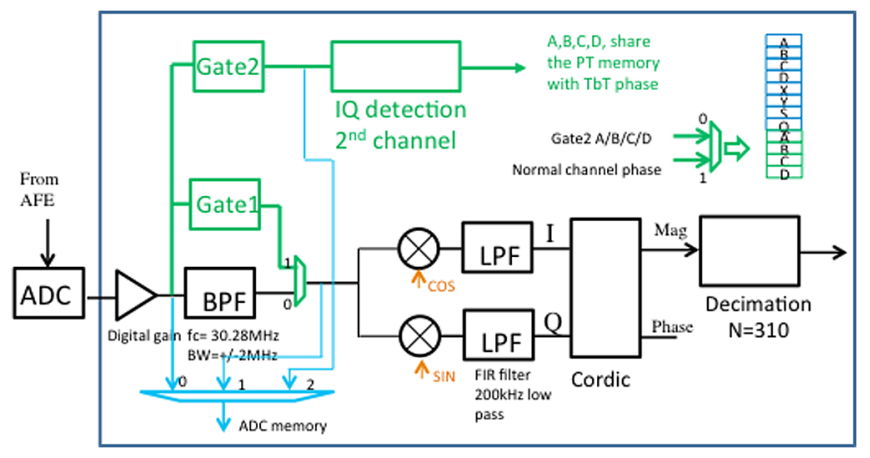

FIG. 4. The schematic diagram of the gated signal processing. Two signal-processing channels with separated gates are provided. One of them (Gate 2) is dedicated to processing the DBT.
Measuring beam positions of only the DBT requires first processing ungated $\mathrm{ADC}$ raw data. Under the filling pattern configuration seen previously in Fig. 1, data are sampled at $117 \mathrm{MHz}$ with 310 samples per turn. The top of Fig. 5 illustrates the ADC raw data from one of the BPM buttons, labeled as "A." The rf attenuation components of the BPM electronics are adjusted with beam current to see the DBT. To get accurate resolution of the DBT, the signals from four buttons ("A-B-C-D") need to be overlapped and their cable delays must be well matched. The gate is then introduced (shown as the red boxes) and the signal processing only includes ADC data sampled from bunches inside the gap as illustrated at the bottom of Fig. 5. Fine timing alignment ( $8 \mathrm{~ns}$ steps) ensures all the BPMs around the ring process the signal from the same bunch(es). After processing the ADC sampled data, the desired beam positions of the DBT can be measured separately from the operational bunch train.

For a short bunch train, the gated $\mathrm{TbT}$ data has better resolution than the ungated TbT data, which can improve the lattice characterization precision. When the storage ring is filled with a short bunch train, most of the samples of the ungated BPM TbT data are taken from empty buckets, which provide no beam signal but contribute to background noise. In contrast, the gated data include about 30 samples, most of which are of nonempty buckets. With the background noise of most of the empty buckets eliminated, the gate function improves the signal-noiseratio $(\mathrm{SNR})$ by a factor of $310 / 30 \approx 10$. The resolution therefore will be $\sqrt{10} \approx 3$ times better than the ungated data. The improved resolution is noticeable in our measurements (see Fig. 6).

Measured TbT data include BPM gain and roll errors, which require calibration before use. For each BPM, four
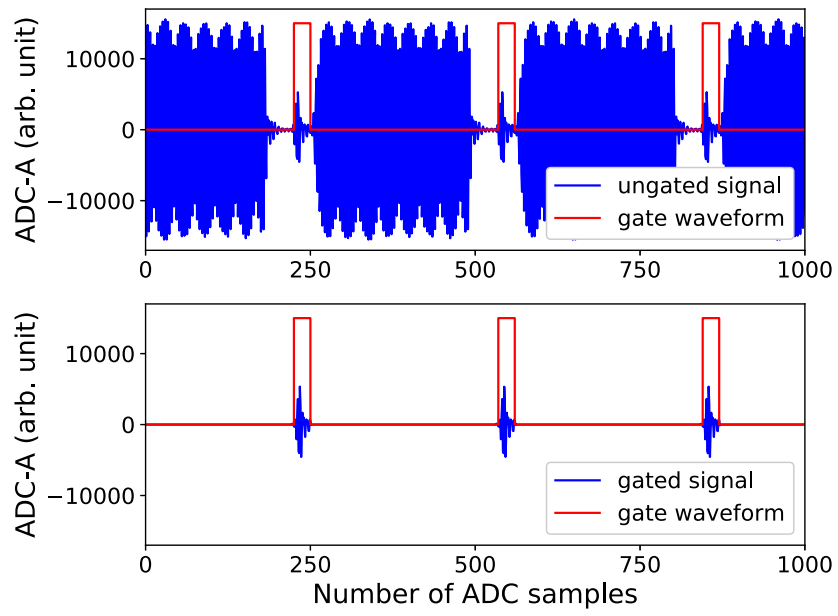

FIG. 5. ADC signal of BPM button "A" with and without gated functionality. Ungated BPM signals are the sum of contributions from all buckets (top). After applying a gated waveform as illustrated by the red lines, the DBT signals can be filtered out and processed by the newly added channel (Gate 2) as seen in Fig. 4. 

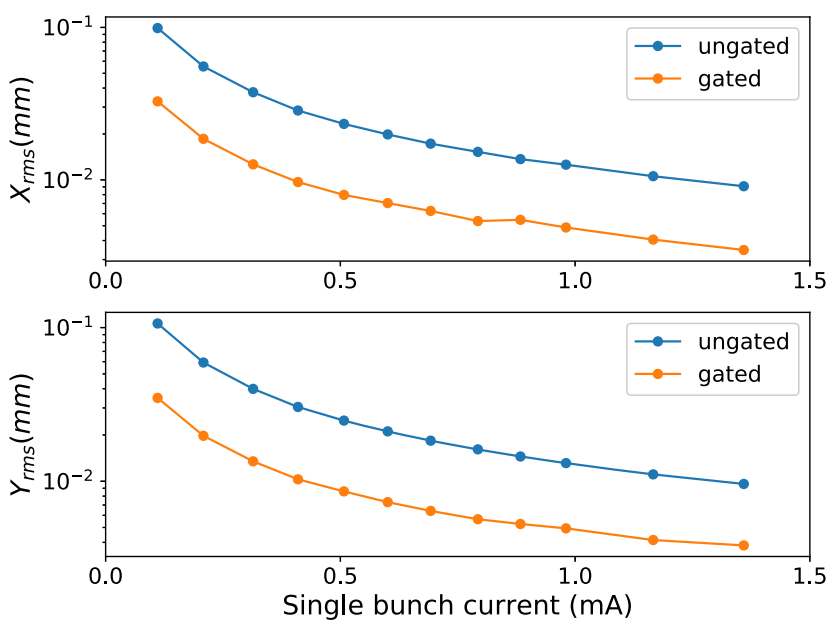

FIG. 6. Comparison of the BPM resolutions for gated and ungated data. Gated BPM data resolution is measured $\sim 3$ times better than the ungated data in both the horizontal and vertical planes.

precalibrated coefficients fitted from a measured orbit response matrix with LOCO [2] are given as

$$
\left(\begin{array}{l}
\bar{x} \\
\bar{y}
\end{array}\right)=\left(\begin{array}{cc}
G_{x} & C_{x} \\
C_{y} & G_{y}
\end{array}\right)\left(\begin{array}{l}
x \\
y
\end{array}\right)
$$

where $(\bar{x}, \bar{y})$ are raw TbT readings, $(x, y)$ are actual beam TbT positions, $G_{x, y}$ are the gain calibrations, and $C_{x, y}$ are the roll calibrations. BPM electronic gains can vary with beam current due to different attenuator settings, but the rolls do not. Betatron phase information, which is independent of BPM gain (see Sec. III), is heavily relied upon in our lattice characterization. The current-dependent gain calibrations therefore have less impact on the data processing. Calibrated TbT data is obtained by implementing the inverse transformation of Eq. (1) on raw data. It is also possible to fit the calibration coefficients from the TbT data directly. As expected, the calibrations from LOCO and TbT data are consistent with each other [19]. The BPMs' gain dependence on the beam current was calibrated and implemented with a field-programmable gate array (FPGA) as well [20]. Experimentally, we used the precalibrated coefficients rather than an on-line fitting which can be more time consuming.

\section{Disturbance of user beam}

Beam disturbance during routine operations is something that all dedicated user facilities strive to minimize. As lattice characterization has traditionally required beam perturbation at levels that would affect users, particularly ones that require high sensitivity, it is important to note the impact that our technique has on the beam stability. To obtain the sufficient resolution for lattice characterization, the DBT amplitude is excited to a maximum of $\sim 1 \mathrm{~mm}$ in our case. The disturbance averaged over all bunches as seen

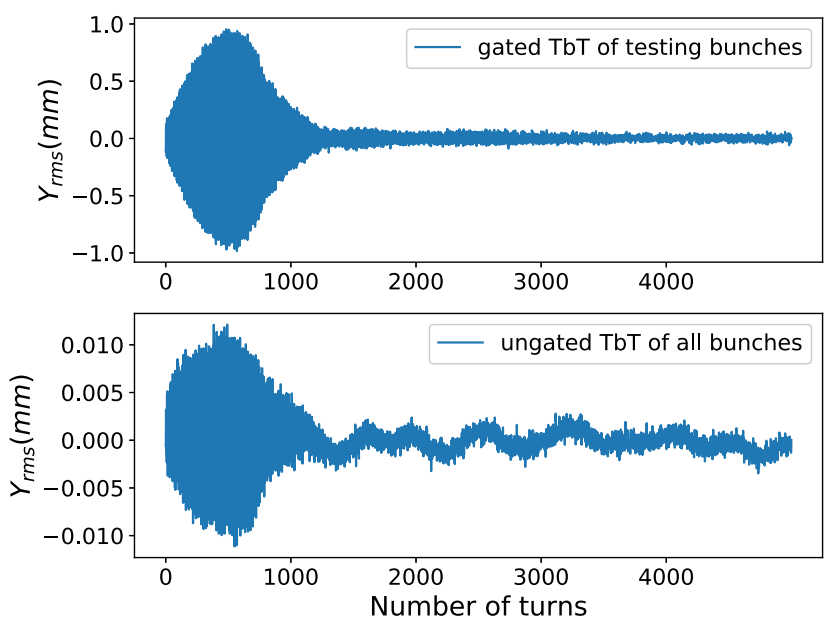

FIG. 7. Comparison of the gated BPM TbT data of the DBT (top) and the ungated $\mathrm{TbT}$ data averaged over all filled buckets (bottom). Although the DBT amplitude reaches $1 \mathrm{~mm}$ for a few $\mathrm{ms}$, the disturbance on the global beam stability is negligible. The ungated data of the combined bunch trains sees noise from either the power supplies or the rf cavities. Note that the two subplots' vertical scales are different.

by the ungated BPM signal, however, is only $1 \%$ or $10 \mu \mathrm{m}$ (see Fig. 7). The $10 \mu \mathrm{m}$ centroid oscillation can be damped within a few ms. It should also be noted that the negligible disturbance created by excitation of the DBT can be triggered on demand, making this measurement transparent to users, even ones with highly sensitive equipment.

\section{LATTICE CHARACTERIZATION METHODS}

This section briefly introduces two methods used to characterize the linear lattice from TbT data at NSLS-II. There are other methods available as well, such as independent component analysis (ICA) [7], which can be used for the same purpose, but are not covered here.

\section{A. Orthogonal decomposition analysis}

The approach of orthogonal decomposition of beam $\mathrm{TbT}$ motion has been proposed before by Castro-Garcia [4]. In the absence of damping and decoherence, and after eliminating the contribution from the closed orbit, the $\mathrm{TbT}$ betatron oscillation as seen by a BPM is

$$
x_{i}=A \sqrt{\beta_{x}(s)} \cos \left[2 \pi \nu_{x} \cdot i+\psi_{x}(s)\right] .
$$

Here, $s$ is the location of the BPM, $i \in[0, N-1]$ is the index of consecutive turns, and $x_{i}$ is the reading of the BPM at the $i$ th turn. $A$ is a constant determined by the initial condition, $\beta_{x}(s)$ and $\psi_{x}(s)$ are the betatron envelope function and phase at the location of $s$, and $\nu_{x}$ is the betatron tune per turn.

Computing two orthogonal modes of the harmonic $\omega_{x}=2 \pi \nu_{x}$ yields 


$$
\begin{aligned}
& C=\sum_{i=0}^{N-1} x_{i} \cos \left(2 \pi \nu_{x} \cdot i\right) \\
& S=\sum_{i=0}^{N-1} x_{i} \sin \left(2 \pi \nu_{x} \cdot i\right) .
\end{aligned}
$$

The amplitude $A \sqrt{\beta_{x}}$ and phase $\psi_{x}$ can be obtained after some algebraic manipulation,

$$
A \sqrt{\beta_{x}}=\frac{2 \sqrt{C^{2}+S^{2}}}{N}, \quad \psi_{x}=-\tan ^{-1}\left(\frac{S}{C}\right),
$$

where the quadrant of the phase $\psi_{x}$ can be determined by the signs of $C$ and $S$. After determining the constant $A$ by scaling the measured $A^{2} \beta_{x}$ with the design $\beta_{x, 0}$, a measured $\beta_{x}$ can be obtained. In Eq. (4), the phase measurement is independent of the BPM gain calibration, which ensures an accurate characterization of the ring lattice.

The error of the amplitude measurement $A \sqrt{\beta_{x}}$ and the phase $\psi_{x}$ from the BPM noise $\sigma$ can be calculated as follows:

$$
\sigma_{A \sqrt{\beta_{x}}}=\sqrt{\frac{2}{N}} \sigma, \quad \sigma_{\psi_{x}}=\frac{1}{A \sqrt{\beta_{x}}} \sqrt{\frac{2}{N}} \sigma .
$$

Equation (5) reveals that in order to achieve a higher precision lattice measurement there are three options: (1) reduce noise error from the BPMs, (2) use data obtained from more turns, or (3) apply a large excitation in measuring the betatron phase. Due to the strong radiation damping and the strong nonlinearity of the NSLS-II ring, options 2 and 3 are not always feasible. In practice, the excitation amplitude within the linear region $(<1 \mathrm{~mm})$ should be controlled, and a reasonable number of turns $(1,000-2,000)$ should be used to get reliable and reproducible measurements. The gated TbT data acquisition technique utilized by our method, however, improves the BPM resolution 3 times better than ungated one, making option 1 the most practical to implement in this case. The statistical results of the multiple snapshots indicate that the error bars of measured $\beta, \psi$ with the gated data are 3 times smaller than with the ungated data which is consistent with our expected result.

\section{B. Principal component analysis}

Principal component analysis (PCA) or model independent analysis was proposed by Irwin and Wang [5,6,21]. For our purposes, Eq. (2) can be rewritten with two subscripts as follows:

$$
x_{j, i}=A \sqrt{\beta_{j}} \cos \left(2 \pi \nu \cdot i+\psi_{j}\right) .
$$

Here subscript $j$ is the azimuthal index of the BPMs along the ring, and $i$ is the temporal index of each individual
BPM's TbT readings. In this notation $x_{j, i}$ is the $i$ th turn reading of the $j$ th BPM. Each $\mathrm{TbT}$ reading can therefore be decomposed as a linear combination of the products of the azimuthal component and the temporal component. $x_{j, i}$ then becomes

$$
\begin{aligned}
x_{j, i} & =A \sqrt{\beta_{j}} \sin \left(\psi_{j}\right) \cos (2 \pi \nu \cdot i)+A \sqrt{\beta_{j}} \cos \left(\psi_{j}\right) \sin (2 \pi \nu \cdot i) \\
& =u_{1} \lambda_{1} v_{1}+u_{2} \lambda_{2} v_{2},
\end{aligned}
$$

where $\lambda_{1,2}=A$ are constants, $u_{1}=\cos (2 \pi \nu \cdot i)$ and $u_{2}=$ $\sin (2 \pi \nu \cdot i)$ are the temporal components, and $v_{1}=$ $\sqrt{\beta_{j}} \sin \left(\psi_{j}\right)$ and $v_{2}=\sqrt{\beta_{j}} \cos \left(\psi_{j}\right)$ are the azimuthal components.

Consider a ring with a total of $M$ BPMs distributed azimuthally. An $N$-turns TbT data array can be constructed as a $2 \mathrm{D} M \times N$ matrix

$$
X_{M, N}=\left[\begin{array}{cccc}
x_{1,1} & x_{1,2} & \cdots & x_{1, N} \\
x_{2,1} & x_{2,2} & \cdots & x_{2, N} \\
\vdots & \vdots & \ddots & \vdots \\
x_{M, 1} & x_{M, 2} & \cdots & x_{M, N}
\end{array}\right]
$$

It can be decomposed as the azimuthal matrix and the temporal matrix in the same way as Eq. (7):

$$
\begin{aligned}
X_{M, N}= & {\left[\begin{array}{cccc}
u_{1,1} & u_{1,2} & \cdots & u_{1, M} \\
u_{2,1} & u_{2,2} & \cdots & u_{2, M} \\
\vdots & \vdots & \ddots & \vdots \\
u_{M, 1} & v_{M, 2} & \cdots & v_{M, M}
\end{array}\right]\left[\begin{array}{cccc}
\lambda_{1} & 0 & \cdots & 0 \\
0 & \lambda_{2} & \cdots & 0 \\
\vdots & \vdots & \ddots & \vdots \\
0 & 0 & \cdots & 0
\end{array}\right] } \\
& \times\left[\begin{array}{cccc}
v_{1,1} & v_{1,2} & \cdots & v_{1, N} \\
v_{2,1} & v_{2,2} & \cdots & v_{2, N} \\
\vdots & \vdots & \ddots & \vdots \\
v_{N, 1} & v_{N, 2} & \cdots & v_{N, N}
\end{array}\right] \\
= & U_{M \times M} S_{M \times N} V_{N \times N}^{T} .
\end{aligned}
$$

Once decomposed in this fashion, it is actually the singular value decomposition of the TbT data array. The azimuthal matrix $V^{T}$ then includes the lattice envelope function $\beta$ and phase $\psi$, which can be extracted as

$$
\begin{aligned}
A \sqrt{\beta_{x, i}} & =\sqrt{\left(\lambda_{1} v_{1, i}\right)^{2}+\left(\lambda_{2} v_{2, i}\right)^{2}} \\
\psi_{x, i} & =\tan ^{-1}\left(\frac{\lambda_{1} v_{1, i}}{\lambda_{2} v_{2, i}}\right) .
\end{aligned}
$$

Typically the first two largest singular values represent the principal component of betatron motion in a specific plane. The next several proceeding singular values 
represent either the linear coupling or the dispersive information. Bad BPMs, if present, can be easily identified as outliers and justifiably removed from the data pool. Some singular values can be identified as specific sources such as the $\sim 60 \mathrm{~Hz}$ noise seen previously in Fig. 7. The remaining small singular values, which cannot be identified as any known error, can be regarded as the BPM background noise. The resolution of measurement can be estimated by summing over the noise signals. The BPM noise can also be eliminated by reconstructing the $\mathrm{TbT}$ array after zeroing down those remaining small singular values.

PCA has many other useful applications such as determining the linear coupling [22], or the wakefields [23], which are not covered here.

\section{Lattice correction strategy}

Once the lattice functions $(\beta, \psi)$ have been characterized, we can compare them with the design model. The distortion can be corrected iteratively with the linear response matrix between $\beta, \psi$ and the focusing strength of the quadrupoles [7]:

$$
\left(\begin{array}{c}
w_{\beta} \Delta \beta \\
w_{\psi} \Delta \psi
\end{array}\right)=\left(\begin{array}{c}
w_{\beta} \mathbf{M}_{\beta} \\
w_{\psi} \mathbf{M}_{\psi}
\end{array}\right)\left(\begin{array}{c}
\Delta K_{1} \\
\Delta K_{2} \\
\vdots \\
\Delta K_{q}
\end{array}\right) .
$$

Here $\Delta \beta=\beta_{\text {meas }}-\beta_{\text {model }}$ are the $\beta$-beats as seen at the locations of BPMs, $\Delta \psi=\psi_{\text {meas }}-\psi_{\text {model }}$ are the phase beats and $w_{\beta, \psi}$ are the weights to balance the $\beta$-beat and phase-beat correction. $\mathbf{M}_{\beta, \psi}=\frac{\partial(\beta, \psi)}{\partial K}$ are the response matrices of the beta and the phase depending on quadrupole strength. $K_{i}=\frac{1}{B \rho}\left(\frac{\partial B_{y}}{\partial x}\right)_{i}, i=1,2, \ldots$ is the $i$ th quadrupole's strength normalized with the beam rigidity $B \rho$.

The response matrices $\mathbf{M}_{\beta, \psi}$ can be calculated from the lattice model. Owing to BPM gain errors, radiation damping and chromatic decoherence, the betatron phase $(\psi)$ can be measured more precisely than the betatron envelope $\beta$ function. In practice, a large weight is assigned to the phase, $\psi$ correction. During early commissioning of NSLS-II, the ring had large distortion which made this particularly necessary.

\section{APPLICATIONS}

In the past at NSLS-II, the lattice was optimized with ungated TbT data of a short bunch train at a low current ( $N_{\text {bunch }} \leq 50, I_{b} \leq 2 \mathrm{~mA}$ ) excited by the pingers. After lattice optimization, a long bunch train of 1,000 buckets, and a dedicated diagnostics Camshaft bunch (a single bunch separated from the main bunch train by 200 empty buckets) were filled and became the standard for routine operations. Under this configuration, the real-time tune of

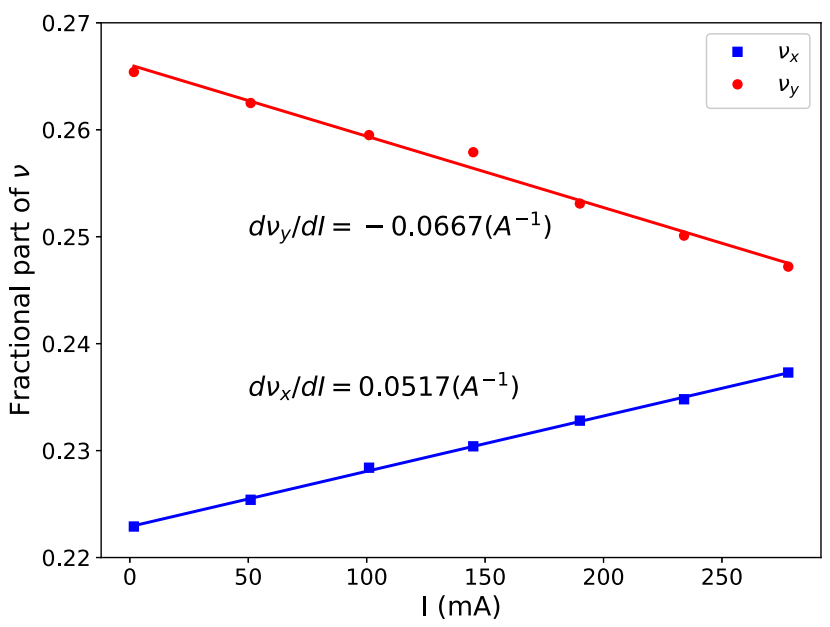

FIG. 8. Tune shifts with stored beam current.

the storage ring can be measured by sweeping betatron frequency on the Camshaft bunch. During beam accumulation, tune drifting has been observed, corresponding to the beam current (Fig. 8). While injecting to higher operations currents, the injection efficiency drops off as well. Although the tune drift can be monitored and corrected, it is typically not possible to localize the distribution of quadrupolar wakefields. To correct the tune under these circumstances requires blind selection of arbitrary quadrupoles to bring the tune back to the nominal value. Blind tune correction such as this often results in extra $\beta$-beat and phase beat.

To measure the lattice drifting with the beam currents, a low charge DBT was injected into the ring. Gated BPM TbT data was then used to measure the lattice function and the correction algorithm from Eq. (11) was applied iteratively to reach a set of optimal magnet settings. For such a low beam current, the effect of the wakefields is negligible. The lattice is solely determined by the external magnetic fields and we refer to the measured lattice under these conditions as the reference lattice. A long bunch train with 1,000 bunches, which is used for operations, was then filled. Instead of filling a Camshaft bunch as has become our standard operating procedure, however, we filled a tenbunch train in place of the Camshaft bunch. The total charge inside the diagnostic train was maintained at a level of about $1 \%$ of the total charge during beam accumulation. At different beam currents, the DBT was selectively excited and its gated TbT data was collected by all 180 BPMs around the ring for lattice characterization. When the stored beam was above $\sim 50 \mathrm{~mA}$, the beam became unstable. It was then necessary to have the BBFB system act on the main operational bunch train to suppress its instabilities.

From the gated BPM TbT data at different currents, we found the lattice was distorted gradually by the wakefields. Tune shift, $\beta$ and $\psi$-beat relative to the stored beam currents are illustrated in Figs. 8 and 9. The tune dependence on the 

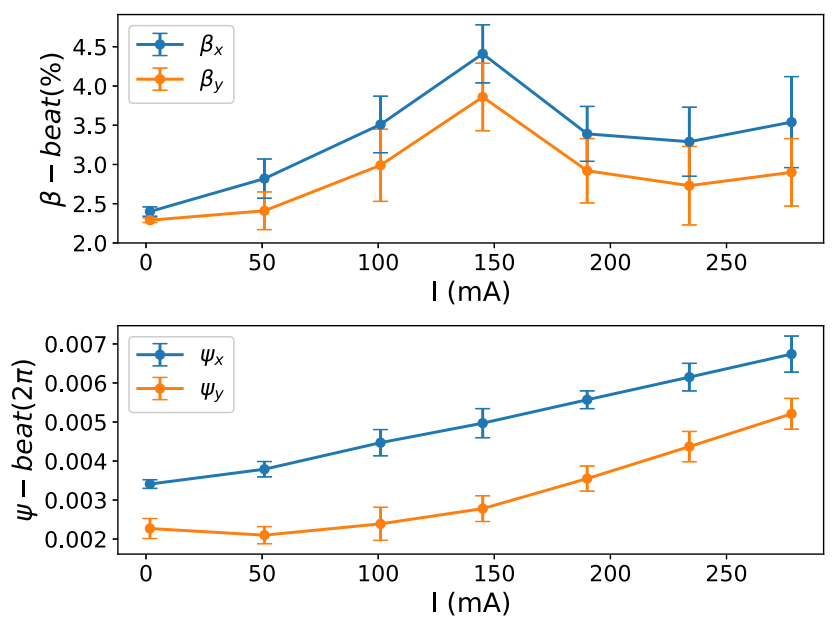

FIG. 9. $\beta$-beat (top) and phase beat (bottom) in relation to stored beam currents.

beam current has contributions from both dipolar and quadrupolar wakefields. Dipolar fields shift tunes in both planes negative, while quadrupolar fields shift tunes in both planes in opposite directions. From Fig. 8 we see that the horizontal and the vertical tunes drift in opposite directions, and the vertical tune shifts faster than the horizontal one. Based on this, it seems most likely that the lattice distortion is mainly due to the quadrupolar wakes of the long-range wakefields of the noncircular vacuum chambers with finite resistivity [24]. The tune shift due to the short range wakefield measured with a single bunch was reported in Ref. [25].

In Fig. 9 we observed gradually developing $\beta$-beat and phase beat around the ring. It is interesting to note that the $\beta$-beat does not increase monotonically with the beam current, but the phase beat does. The large $\beta$-beat at $150 \mathrm{~mA}$ comes from several local $\beta$-bumps at large $\beta$ sections, where the phase beat $(\alpha 1 / \beta)$ is not so sensitive. The phase information of betatron oscillation can be measured more precisely than the envelope function $\beta$, therefore our lattice corrections rely heavily on the phase measurement in Eq. (11).

Based on the measured $\beta$ and $\psi$-beats we can use Eq. (11) to locate the quadrupolar wakefields by putting numerous virtual quadrupoles around the ring. In Fig. 10, the variation of the vertical phase advance as a function of beam current is shown around the location of a $7 \mathrm{~m}$ long damping wiggler, which has a flat chamber. In reality we need to use real quadrupoles to compensate for the wakefields. The distribution of the needed compensation $\Delta K_{i}$, as well as the variation of phase advance with beam current, can therefore be used to approximate the locations of impedance. The importance of localizing the wakefields of vacuum chambers becomes apparent from this analysis. During characterization of the NSLS-II storage ring, multiple locations were discovered where the needed

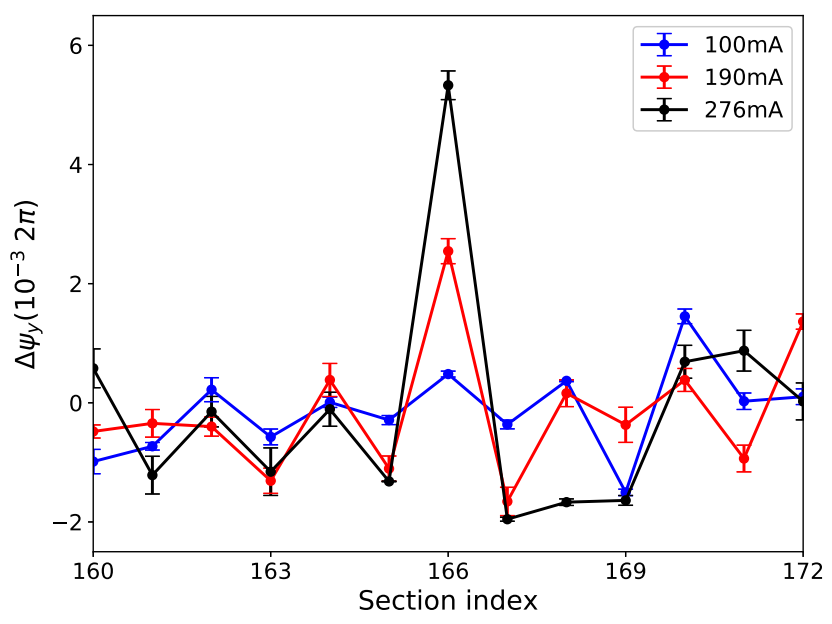

FIG. 10. Variation of the vertical phase advance as a function of beam current around the location of a $7 \mathrm{~m}$ long damping wiggler (section 166), which has a flat chamber.

quadrupole compensation was gradually increasing with the stored beam current (see Fig. 11). Consequently, the integrated quadrupole strength which is used to compensate for the quadrupolar wakefields for the whole ring is also found linearly scaled with beam currents (see Fig. 12). An alternative and possibly better method for localizing the wakefields would be to utilize the TbT data of the virtual quadrupole's neighboring BPMs. This data can be used to calibrate the strength of the wakefields which might be able to minimize the degeneracy from its neighboring quadrupoles $[3,26]$.

Tune drifts that correspond to beam current have been observed previously in several other high-energy storage rings and therefore are well understood [24,27]. The distributed wakefields were localized at other machines

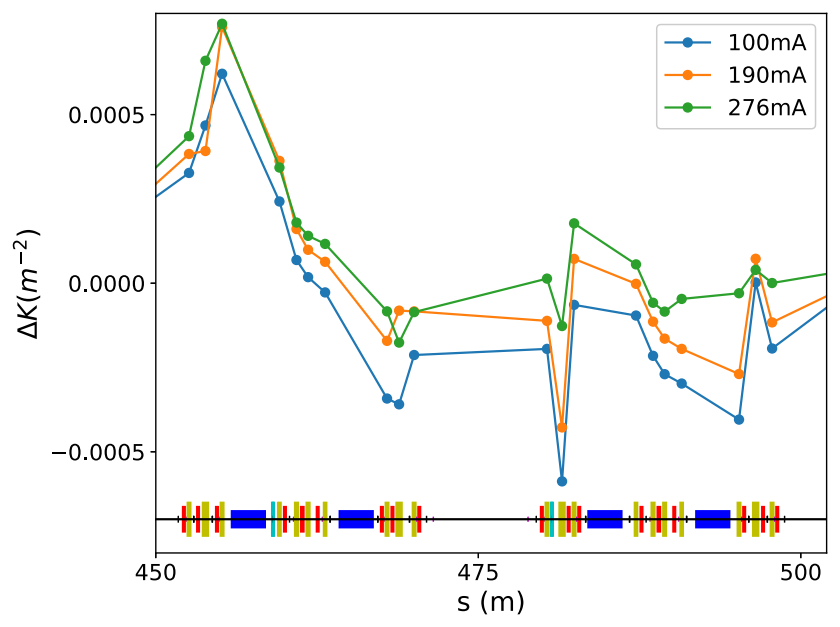

FIG. 11. Quadrupoles' virtual variations at different beam currents obtained with Eq. (11) for a NSLS-II supercell. They can be interpreted as horizontal focusing quadrupolar wakefields that gradually increase with the beam current, which provides a guide for localizing the impedance distribution around the ring. 


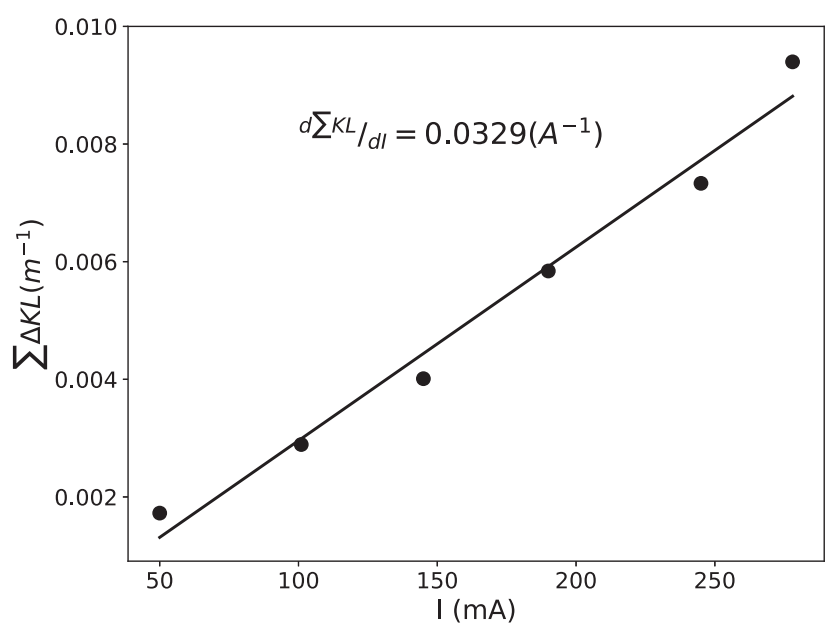

FIG. 12. Integrated quadrupole strength which is needed to compensate for the quadrupolar wakefields at different beam currents.

during dedicated beam study time [28,29]. With the advent of our method, it is now possible to measure not only the incoherent tune drifts, but also the distributed $\beta$-beat and phase beat around the storage ring during user operation. A systematic correction strategy can therefore be implemented to mitigate distortion of the linear lattice. The tune drift only represents the integrated distorted optics over the whole ring, but the distributed $\beta$-beat and phase beat enable us to identify and localize potential flaws in the vacuum structure, which can generate large impedance. At NSLS-II, the localized quadrupolar wakefields are mostly distributed evenly due to the flat dipole and insertion device chambers.

The resulting tune drifts in the horizontal and the vertical planes due to the quadrupolar wakefields' have opposite signs. The ring's working point of $\nu_{x / y}=33.22 / 16.26$, therefore, approaches a linear resonance line during beam accumulation. During beam accumulation the horizontal beam emittance does not deviate significantly. The convergence of the fractional parts of $\nu_{x}$ and $\nu_{y}$ can significantly increase the vertical emittance and degrade the brightness (see Fig. 13). With the TbT data we can reconstruct the coupled linear one-turn-matrix $\mathbf{R}(s)$ at the locations of the BPMs [30]. Here we used MaisRipken's parametrization [31-33] to interpret the measured $\mathbf{R}(s)$. Aside from two dominating envelope functions $\beta_{x, I}$ and $\beta_{y, I I}$, there exist two other small $\beta_{x, I I}$ and $\beta_{y, I}$, which represent the coupling strength between two planes. The comparison of measured coupling $\beta$ s at two significantly different beam currents is illustrated in Fig. 14. In this scenario, the tunes measured before are not the actual pure horizontal or vertical oscillation frequencies. They belong to one of two coupled modes $I, I I$ respectively. The skew quadrupole components that increase the vertical emittance mainly come from the tilted ring quadrupoles, the vertical

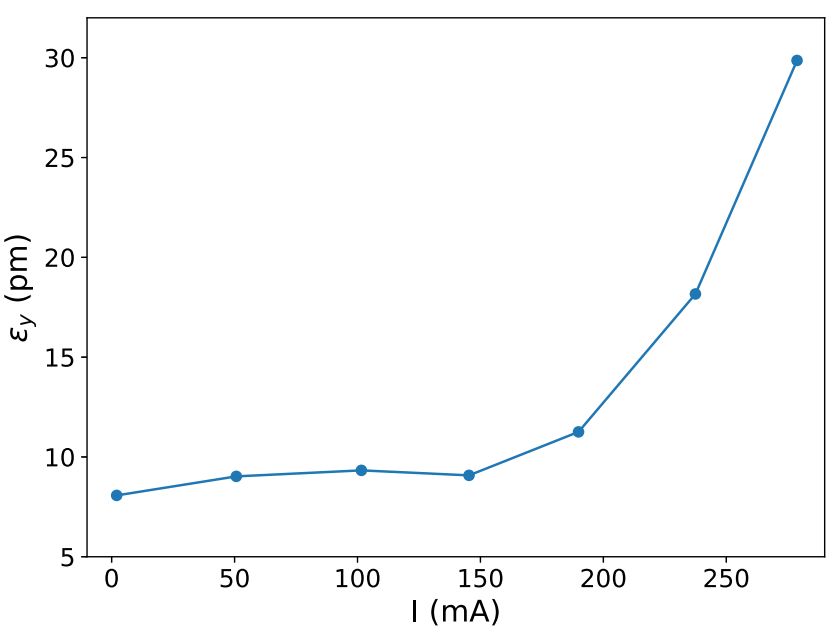

FIG. 13. Vertical emittance is drastically increased when the tune approaches a linear resonance during beam current accumulation.

orbit displacement in the sextupoles and the imperfection of insertion devices.

The dynamic aperture of modern storage rings is highly sensitive to the phase advance among the sextupoles. At NSLS-II, $10 \%-15 \%$ decrease in injection efficiency was frequently observed at the current nominal operating current (275-300 mA). To understand that, a simulation code, ELEGANT [34], was used to simulate the distorted lattice dynamic aperture. The simulation involved adding the corresponding quadrupole strength adjustments on top of the external quadrupole settings directly. The dynamic aperture was found to decrease gradually with increasing beam current if no linear lattice correction was implemented (see Fig. 15). Injection efficiency can sometimes be restored more or less by blindly moving the horizontal and vertical tunes back to their nominal values. The lattice distortion, however, cannot be restored in this fashion.

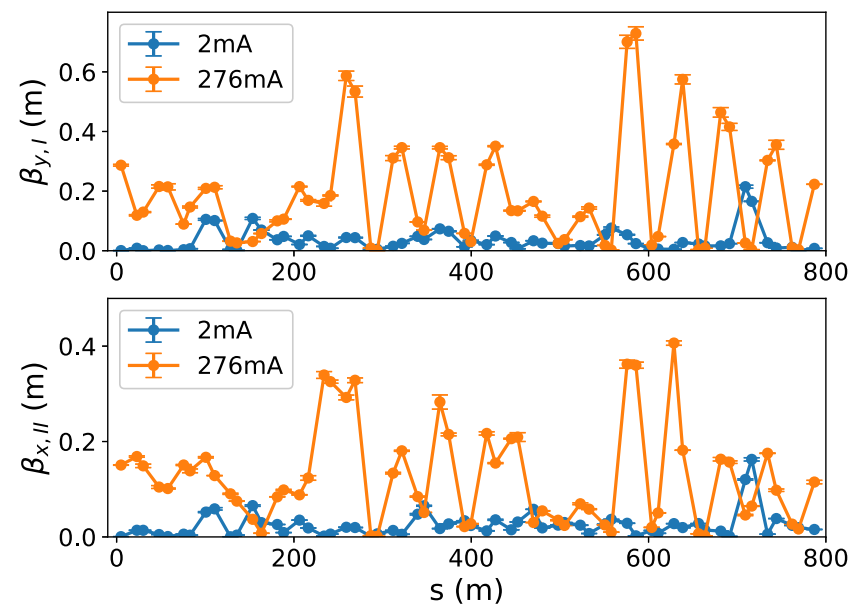

FIG. 14. Comparison of two coupling $\beta$-functions at a low beam current $(2 \mathrm{~mA})$ and at a higher beam current $(276 \mathrm{~mA})$. 


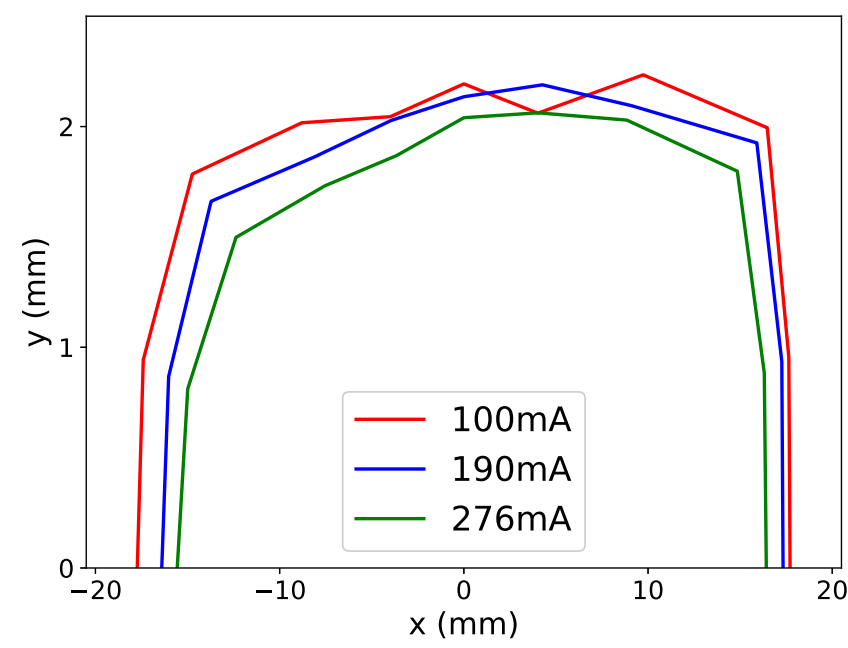

FIG. 15. Dynamic aperture reduction at different stored beam currents without linear lattice correction at NSLS-II. In addition to the quadrupole nominal settings, localized quadrupolar wakefields are introduced in the lattice. The systematic and random multipole errors, closed orbit distortion and linear coupling are included in the simulations. Each dynamic aperture is obtained, accounting for random multipole error distributions in specific magnets, and averaged over 50 random seeds.

If the lattice continues to become more distorted by repeatedly adjusting the tunes blindly, it could result in a reduction of the local energy acceptance as well as the Touschek lifetime. NSLS-II operates in top-off mode to maintain a steady beam current during operations. The frequency of top-off shots is therefore dependent on the beam lifetime. A shorter lifetime means more frequent topoff shots and less stability to the beam lines. From the point of view of a user facility, an optimized lattice at operational conditions is critical to achieving peak performance. Our method allows us to monitor and compensate the distorted linear lattice transparently during routine operation.

After accumulating $\sim 275 \mathrm{~mA}$ in the NSLS-II storage ring, the lattice distortion was mitigated with 300 independently powered quadrupoles. A lattice parameters comparison at two beam currents is illustrated in Table II. The $\beta$-beat and phase beat after correction was worse than at $2 \mathrm{~mA}$, but was significantly improved compared to the uncorrected lattice. Lattice distortion cannot be completely eliminated, owing to the quadrupolar wakefields generated at locations of noncircular vacuum chambers, such as dipole chambers. While the quadrupoles used for lattice compensation are not located at the exact points of measurement, the lattice distortion can only be mitigated to a certain extent. Presently, NSLS-II operates at a stored beam current of $300 \mathrm{~mA}$. Even at such a current the lattice distortion due to the quadrupolar wakefields is tolerable after a blind tune correction. The goal at NSLS-II is to eventually operate at top-off current of $500 \mathrm{~mA}$. At this elevated current, lattice distortion can be expected to be greater and may not be as tolerable to blind tune
TABLE II. Comparison of measured lattice distortions at different beam currents.

\begin{tabular}{lccc}
\hline \hline$I_{b}(\mathrm{~mA})$ & 2 & $276^{\mathrm{a}}$ & $276^{\mathrm{b}}$ \\
\hline$\nu_{x}$ & 33.223 & 33.237 & 33.225 \\
$\nu_{y}$ & 16.265 & 16.247 & 16.260 \\
$(\Delta \beta / \beta)_{x}(\%)$ & $2.4 \pm 0.06$ & $3.5 \pm 0.58$ & $2.7 \pm 0.29$ \\
$(\Delta \beta / \beta)_{y}(\%)$ & $2.3 \pm 0.03$ & $2.9 \pm 0.43$ & $2.6 \pm 0.46$ \\
$\Delta \psi_{x}\left(2 \pi \times 10^{-4}\right)$ & $34.1 \pm 1.1$ & $67.4 \pm 4.6$ & $45.2 \pm 2.9$ \\
$\Delta \psi_{y}\left(2 \pi \times 10^{-4}\right)$ & $22.7 \pm 2.6$ & $52.1 \pm 4.0$ & $20.8 \pm 4.3$ \\
$\epsilon_{y}(p m)$ & 8.07 & 29.8 & 8.46 \\
\hline \hline
\end{tabular}

${ }^{\mathrm{a} B e f o r e}$ correction.

${ }^{\mathrm{b}}$ After correction.

corrections. It would therefore be prudent to implement a real-time lattice monitoring and correction program by scaling the wakefields with the beam current linearly.

\section{SUMMARY}

NSLS-II is a premiere third generation synchrotron light source and a state-of-the-art research facility. It is therefore paramount that performance of the accelerator is optimal. A key component of optimizing performance is minimizing linear lattice distortion. Traditional methods of lattice characterization have been intrusive during routine operations. We offer an improved, transparent approach. Our method applies selective gated transverse excitation and data acquisition of a small DBT. The distributed lattice distortion can be extracted from the DBT excitation data and a systematic correction applied to mitigate it. Our approach overcomes several difficulties faced by traditional methods; common ones being interruption or disturbance of beam line experiments, or characterizing and correcting distortion from dynamic sources such as wakefields generated in vacuum chambers.

Although the BPM system electronics at NSLS-II can be configured to resolve the signals of two well-separated bunch trains, some short or medium range wakefields, such as fast-ion wake [35], actually lead to tiny differences among all the bunches. Lattice characterization and correction has been implemented based on the DBT thus far, however, it might not be an optimal solution for the main operational bunch train. NSLS-II in-house developed BPM electronics, as well as the widely used commercial product [36], use $125 \mathrm{MHz}$ ADC that were designed over ten years ago. With their 14-bit ADCs, $125 \mathrm{MHz}$ sampling rate was state-of-the-art at that time. A lower sampling rate makes the following FPGA signal processing easier to handle as well. But for storage rings equipped with a $500 \mathrm{MHz} \mathrm{rf}$ system, it is impossible to resolve the bunch-to-bunch signals we are concerned with. In this case, it will be necessary to develop a high resolution bunch-by-bunch BPM electronics system [37] to further improve diagnostics capabilities and therefore optimize machine performance. 


\section{ACKNOWLEDGMENTS}

We would like to thank our NSLS-II colleagues for supporting this study. This work was supported by Department of Energy Contract No. DE-SC0012704.

W. C. and Y. L. contributed equally to this work.

[1] BNL, https://www.bnl.gov/nsls2/project/PDR/.

[2] J. Safranek, Experimental determination of storage ring optics using orbit response measurements, Nucl. Instrum. Methods Phys. Res., Sect. A 388, 27 (1997).

[3] X. Huang, J. Safranek, and G. Portmann, LOCO with constraints and improved fitting technique, ICFA Beam Dynamics Newsletter 44, 60 (2007).

[4] P. Castro-Garcia, Ph.D. thesis, CERN, 1996.

[5] J. Irwin, C. X. Wang, Y. T. Yan, K. L. F. Bane, Y. Cai, F. J. Decker, M. G. Minty, G. V. Stupakov, and F. Zimmermann, Model-Independent Beam Dynamics Analysis, Phys. Rev. Lett. 82, 1684 (1999).

[6] C. X. Wang, Ph.D. thesis, Stanford University, 1999.

[7] X. Huang, S. Lee, E. Prebys, and R. Tomlin, Application of independent component analysis to Fermilab Booster, Phys. Rev. ST Accel. Beams 8, 064001 (2005).

[8] R. Tomás, M. Aiba, A. Franchi, and U. Iriso, Review of linear optics measurement and correction for charged particle accelerators, Phys. Rev. Accel. Beams 20, 054801 (2017).

[9] A. Franchi, Error analysis of linear optics measurements via turn-by-turn beam position data in circular accelerators, arXiv:1603.00281.

[10] G. Rehm, M. Abbott, A. Morgan, J. Rowland, and I. Uzun, 14th beam instrumentation workshop (2010).

[11] R. E. Meller, A. W. Chao, J. M. Peterson, S. G. Peggs, and M. Furman, Report No. SSC-N-360, 1987.

[12] S. Y. Lee, Report No. SSC-N-749, 1991.

[13] I. Karpov, V. Kornilov, and O. Boine-Frankenheim, Early transverse decoherence of bunches with space charge, Phys. Rev. Accel. Beams 19, 124201 (2016).

[14] P. Lebasque, R. B. E. Fekih, M. Bol, J. P. Lavieville, A. Loulergue, and D. Muller, Conf. Proc. C0806233, WEPC081 (2008).

[15] W. Cheng, Y. Li, and K. Ha, Techniques for transparent lattice measurement and correction, J. Phys. Conf. Ser. 874, 012082 (2017).

[16] W. Cheng, B. Bacha, D. Teytelman, Y. Hu, H. Xu, and O. Singh, NSLS2 Diagnostic Systems Commissioning and Measurements, in Proceedings of IBIC2014, Monterey, CA, 2014 (2014), p. 707.

[17] Dimtel Inc., http://www.dimtel.com.

[18] K. Vetter et al., Conf. Proc. C110328, 495 (2011).
[19] Y. Hidaka and X. Yang (private communication).

[20] W. Cheng, B. Bacha, D. Padrazo, J. Mead, M. Maggipinto, K. Ha, and Y. Hu, in Proceedings of IBIC2014, Monterey, CA, 2014 (2014), p. 16.

[21] C. X. Wang, V. Sajaev, and C. Y. Yao, Phase advance and $\beta$ function measurements using model-independent analysis, Phys. Rev. ST Accel. Beams 6, 104001 (2003).

[22] C. X. Wang, Untangling mixed modes in modelindependent analysis of beam dynamics in circular accelerators, Phys. Rev. ST Accel. Beams 7, 114001 (2004).

[23] Z. Chen, Y. Yang, Y. Leng, and R. Yuan, Wakefield measurement using principal component analysis on bunch-by-bunch information during transient state of injection in a storage ring, Phys. Rev. ST Accel. Beams 17, 112803 (2014).

[24] A. Chao, S. Heifets, and B. Zotter, Tune shifts of bunch trains due to resistive vacuum chambers without circular symmetry, Phys. Rev. ST Accel. Beams 5, 111001 (2002).

[25] W. Cheng, B. Bacha, D. Teytelman, Y. Hu, H. Xu, and O. Singh, in Proceedings of IBIC2014, Monterey, CA, 2014 (2014), p. 707.

[26] X. Huang, J. Sebek, and D. Martin, Lattice modeling and calibration with turn-by-turn orbit data, Phys. Rev. ST Accel. Beams 13, 114002 (2010).

[27] P. Brunelle, R. Nagaoka, and R. Sreedharan, Measurement and analysis of the impact of transverse incoherent wakefields in a light source storage ring, Phys. Rev. Accel. Beams 19, 044401 (2016).

[28] D. Brandt, P. Castro, K. Cornelis, A. Hofmann, G. Morpurgo, G. L. Sabbi, J. Wenninger, and B. Zotter, Conf. Proc. C950501, 570 (1996).

[29] V. Sajaev, ICFA Beam Dynamics Newsletter 44, 101 (2007).

[30] Y. Li, L. Yang, and W. Cheng, Characterization and control of linear coupling using turn-by-turn beam position monitor data in storage rings, arXiv:1706.06022.

[31] H. Mais and G. Ripken, DESY Report No. M-82/05, 1982.

[32] F. Willeke and G. Ripken, Methods of beam optics, AIP Conf. Proc. 184, 758 (1989).

[33] V. Lebedev and S. Bogacz, Betatron motion with coupling of horizontal and vertical degrees of freedom, J. Instrum. 5, P10010 (2010).

[34] M. Borland, Advanced Photon Source Report No. LS-287, 2000.

[35] W. Cheng, Y. Li, and B. Podobedov, Experimental evidence of ion-induced instabilities in the NSLS-II storage ring, Nucl. Instrum. Methods Phys. Res., Sect. A 861, 38 (2017).

[36] Instrumentation Technologies d.d., http://www.i-tech.si.

[37] J. Shanks, D. Rubin, and D. Sagan, Low-emittance tuning at the Cornell Electron Storage Ring Test Accelerator, Phys. Rev. ST Accel. Beams 17, 044003 (2014). 